\title{
Si-DOPING OF MOCVD GaAs: CLOSER ANALYSIS OF THE INCORPORATION PROCESS
}

\author{
X. TANG * , H.G.M. LOCHS, P.R. HAGEMAN, M.H.J.M. DE CROON and L.J. GILING \\ Department of Experimental Solid State Physics, RIM, Faculty of Science, University of Nijmegen, Toernooiveld, \\ 6525 ED Nijmegen, The Netherlands
}

and

\author{
A.J. BONS \\ Laboratory for Metallurgy, Delft University of Technology, 2628 CJ Delft, The Netherlands
}

Received 10 July 1989; manuscript received in final form 30 August 1989

\begin{abstract}
Doping studies have been carried out as a function of silane input fraction over the entire dopant range of silicon in GaAs. In the lower dopant region where the incorporation varies linearly with the silane input mole fraction, the compensation ratio $N_{\mathrm{A}}^{-} / N_{\mathrm{D}}^{+}$ appears to have a constant value of 0.3. In this region the incorporation also is orientation dependent. For $\mathrm{SiH}_{4}$ input mole fractions higher than $1 \times 10^{-6}$ the electron concentration does not change noticeably, however the compensation ratio rises from 0.3 to 0.7 . Simultaneously precipitates are observed in the grown layer. From gas phase equilibrium calculations such silicon precipitation is predicted to take place at these higher input pressures of $\mathrm{SiH}_{4}$. As regards the incorporation rate of $\mathrm{Si}$ itself, analysis of the doping results reveals that, when corrections are made for differences in GaAs growth rate and small differences in growth temperature, a constant deposition of silicon along the MOCVD reactor is obtained. The absence of depletion effects and the observed temperature dependence imply that the limiting step for the $\mathrm{Si}$ incorporation is the decomposition of the $\mathrm{SiH}_{4}$ which happens very close to the hot surface of the GaAs substrate.
\end{abstract}

\section{Introduction}

Metalorganic chemical vapour deposition (MOCVD) now has become a standard and versatile technique with a proved capability to grow many sophisticated structures such as superlattices, lasers and tandem solar cells. For most device applications, a good control of the doping process is necessary, including control of doping level, uniformity and doping profile. Up to now, no single dopant seems to be able to fulfill all the requirements such as wide doping range, easy control of uniformity, easy to handle, negligible memory effect, low diffusion coefficient, and so on. As an n-type dopant, silicon is widely used for GaAs. For a long time, silane $\left(\mathrm{SiH}_{4}\right)$ has been

* On leave from: The Department of Applied Physics, Chongqing University, Chongqing, People's Rep. of China. used as the doping source. One of the dominant problems inherent with this dopant is a strong temperature dependence which makes uniform doping difficult for reactors where the temperature differs over the susceptor. When silane is replaced by disilane, the incorporation process appeared to be independent of growth temperature [1,2]. However, recent analysis has revealed that actually disilane should make no difference when compared with silane, except when lower pressures are used or when the flow and temperature profiles of the incoming gas are undeveloped $[3,4]$. Another well recognized effect is the compensation which can occur with silicon. In literature, for low and moderate Si concentrations, this problem is more or less ignored and it is assumed that silicon is incorporated as a donor $[5,6]$. In the present report, the compensation effect is studied over the entire dopant range. Another subject

0022-0248/89/\$03.50 (c) Elsevier Science Publishers B.V.

(North-Holland Physics Publishing Division) 
which has not received much attention is the formation of Si precipitates in the GaAs lattice what occurs at the highest doping levels. This effect has been studied using interference-contrast microscopy, photoluminescence and transmission electron microscopy (TEM), assisted by thermodynamic calculations.

In our long horizontal MOCVD reactor, which enables us to grow with well established flow and temperature profiles, it should in principle also be possible to reveal more about the details of the $\mathrm{Si}$ incorporation. Therefore the study is elaborated by examining the $\mathrm{Si}$ incorporation on various crystallographic orientations.

\section{Experimental}

The doping experiments were carried out at atmospheric pressure using trimethylgallium (TMG) and arsine $\left(\mathrm{AsH}_{3}\right)$ as source materials while $100 \mathrm{ppm}$ silane $\left(\mathrm{SiH}_{4}\right)$ in $\mathrm{H}_{2}$ was employed as dopant source. A horizontal reactor is used with a rectangular cross-section. The reactor is resistance heated at the bottom and water cooled on the top so that a known temperature profile is established. A long entrance length is used to ensure stabilization of both temperature and velocity profiles of the incoming gas mixture (for details, see ref. [7]). The arsine was passed through a molecular sieve before mixing with the carrier gas $\left(\mathrm{H}_{2}\right)$ in order to minimize the moisture level [8]. The ratio between $\mathrm{AsH}_{3}$ and TMG (V/III ratio) was 20 . The growth temperature was measured at the GaAs substrate surface with a calibrated optical pyrometer. It was kept constant at $700 \pm 5^{\circ} \mathrm{C}$ for all the present MOCVD experiments except during the temperature series which was used to determine the activation energy of the $\mathrm{Si}$ incorporation. The GaAs substrates obtained from MCP (UK) were Cr-doped semi-insulating, horizontal Bridgman grown, and chemo-mechanically polished on one face. Typical etch pit densities were below $5000 / \mathrm{cm}^{2}$. The substrate orientations were $(100) 2^{\circ}$ off towards $(110),(110)$, (111) Ga, (111)As, (100) $4^{\circ}$ off towards (110), and (100). The $(100) 2^{\circ}(110)$ substrate is widely used because it is known to give the best morphology.
Also in our experiments this orientation was mainly used. The other orientations $(110),(111) \mathrm{Ga}$, and (111)As have been employed in order to study the orientation dependence of the $\mathrm{Si}$ incorporation process. However it appeared that under our experimental conditions, the growth on the exact (111)As substrate always resulted in a rough surface with correspondingly unreliable Hall mobilities. Therefore these results had to be discarded. The orientations $(100) 4^{\circ}(110)$ and (100) specifically have been used for studying the $\mathrm{Si}$ precipitation.

The morphology after growth was observed with an interference-contrast microscope. The thickness of the grown layer was measured by cleaving and staining. The electrical characterization was performed by Van der Pauw-Hall measurements using a clover-leaf configuration. Photoluminescence measurements were done at $5 \mathrm{~K}$ with an $\mathrm{Ar}^{+}$laser $(514.5 \mathrm{~nm})$ and a double-monochromator fitted with an S1 photomultiplier. TEM measurements were performed to examine the precipitates in the epitaxial layers. All TEM samples have been thinned by mechanical polishing followed by ion beam milling using $\mathrm{Ar}^{+}$ions. The samples were studied in a Philips EM400T TEM operating at $120 \mathrm{kV}$.

\section{Results and discussion}

\subsection{Electrical evaluations}

In fig. 1 the net carrier concentrations $n\left(N_{\mathrm{D}}^{+}-\right.$ $\mathrm{N}_{\mathrm{A}}^{-}$) as a function of the input mole fraction of $\mathrm{SiH}_{4}$ are given for three substrate orientations, viz. $(100) 2^{\circ}(110)$, and the exact orientations (111) Ga and the (110). Below a silane mole fraction of $10^{-6}$, a linear relationship is found for all three substrate orientations. Above this value saturation of the carrier concentration occurs. This is in accordance with literature [9-11]. As is evident from fig. 1 the difference in incorporation on the various substrate orientations is especially clear in the lower dopant regions. The efficiency of the incorporation increases in the order $(100) 2^{\circ}(110)$ $>(111) \mathrm{Ga}>(110)$. This is in agreement with the results of Veuhoff et al. [2] on the Si-doping 


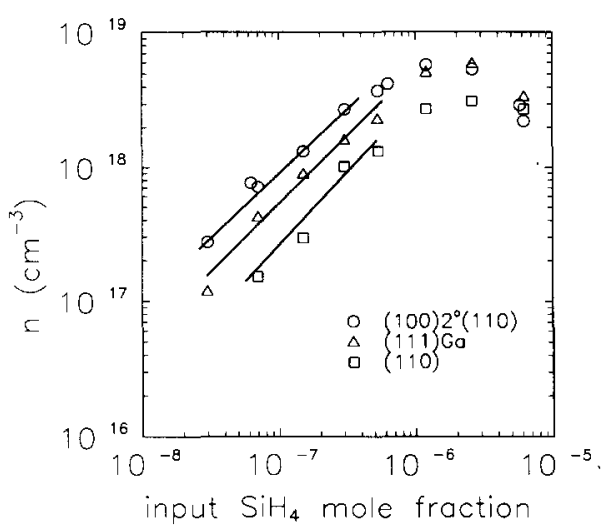

Fig. 1. Net carrier concentration $n=N_{\mathrm{D}}^{+}-N_{\mathrm{A}}^{-}$versus the input mole fraction of silane for three different substrate orientations. $T_{\mathrm{G}}=700^{\circ} \mathrm{C}, \mathrm{V} / \mathrm{III}=20$.

studies of the (111)As, (100) and (111)Ga orientations. Veuhoff et al. explained the difference on the (111)Ga and (111)As surfaces by assuming that on an ideal (111)Ga surface all the dangling bonds are empty, whereas on the (111)As surface each arsenic surface atom possesses a dangling bond filled with a pair of electrons. Because of this difference, adsorption of $\operatorname{SiH}_{x}(x \leq 3)$ radicals should be favoured on the (111)As surface. No explanation was given in that study for the behaviour of the (100) surface. A similar trend is also found by Nakanisi [12] in intentionally undoped GaAs grown by MOCVD where the background impurity concentration for five used orientations increases in the order (311)B $>(100)>$ (111)A $>$ (311) $\mathrm{A}>$ (110). Nakanisi did not give an explanation for the incorporation differences however. A few orientation dependent studies have been made on Si-doped GaAs grown by MBE [13-15]. In these studies the observed differences on A ((211)A, (311)A, (511)A, (711)A) and B ((211)B, (311)B, (511)B, (711)B) polar faces were explained by the assumption of coexistence of a single- and double-dangling bonds from the (111) faces and the (100) face respectively where the bonding between the adatom and the substrate is stronger at the double-dangling bond site than that at the single-dangling bond site. With this assumption they could explain the tendency of the $A$ and $B$ polar faces to be doped as p-type and n-type respectively.
The linear relation between carrier concentration and the silane input concentration, as observed in the lower dopant region, points to a first order reaction as the rate limiting step. The most likely process is subsurface trapping of the incorporated dopant, thereby breaking the exchange between adsorbed dopant atoms and neutral donors in the solid. This will occur whenever the vertical growth rate $(G)$ is faster than the diffusing rate of subsurface $\mathrm{Si}_{\mathrm{Ga}}$ to the surface $(D)$, the actual condition being $G>D / b$, where $b$ is of the order of the lattice constant. This automatically means that the ionization of the dopant cannot reflect itself in the slope of the curve. It must be remembered that when a full equilibrium for the dopant system is present, a slope $1 / 2$ in the figure would be expected. Instead of subsurface trapping, trapping of the adsorbed dopant atoms by the moving steps can be the limiting factor. That step trapping at least partially is active is illustrated in fig. 2 where a comparison is given of the $\mathrm{Si}$ incorporation on $(100)$ orientations with respectively $0^{\circ}, 2^{\circ}$ and $4^{\circ}$ of misorientation. Although on the exact (100) surface two-dimensional nucleation is possible, which will introduce extra steps on the surface, we can still assume that the number of steps will increase with an increase in the degree of misorientation. When the growth rate is the same for all these three orientations,

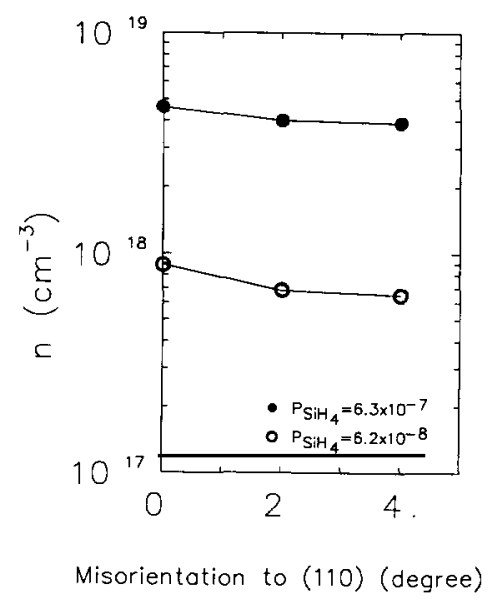

Fig. 2. Net carrier concentration $n=N_{\mathrm{D}}^{+}-N_{\mathrm{A}}^{-}$versus the degree of misorientation of the (100) surface towards (110). $T_{\mathrm{G}}=700^{\circ} \mathrm{C}, \mathrm{V} / \mathrm{III}=20$. 
which is found experimentally, the step velocity should decrease with an increase in misorientation. As a consequence the effect of step trapping will be more effective for samples that are less misoriented, as is observed (fig. 2). It must be remarked here that also the total amount of incorporated silicon (i.e. $\mathrm{Si}_{\mathrm{Ga}}+\mathrm{Si}_{\mathrm{As}}$ ) shows the same dependence on misorientation so that the effect as given in fig. 2 is not influenced by a possible change in compensation.

The room temperature Hall mobilities as a function of carrier concentration are given in fig. 3 . No difference is found between the layers grown on the $(100) 2^{\circ}(110)$ and $(111) \mathrm{Ga}$ orientations. Above carrier concentrations of $3 \times 10^{18} \mathrm{~cm}^{-3}$, the mobility values clearly diverge however from the theoretical $N_{\mathrm{A}}^{-} / N_{\mathrm{D}}^{+}=0.3$ curve. The layers grown on the (110) orientation have overall lower mobility values as compared with the layers grown on the other two orientations.

Because $\mathrm{Si}$ is a group IV element, a $\mathrm{Si}$ atom may be incorporated at a $\mathrm{Ga}$ site which gives rise to a donor, or be incorporated at an As site which gives rise to an acceptor, so that this amphoteric behaviour leads to compensation. The compensation ratio which is defined as $N_{\mathrm{A}}^{-} / N_{\mathrm{D}}^{+}$can be calculated from the measurements of carrier concentration and mobility $[16,17]$. It should be

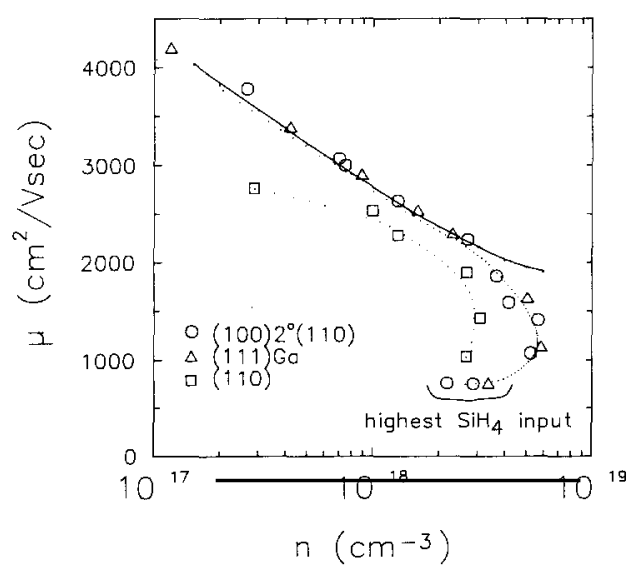

Fig. 3. Hall mobility at room temperature versus the carrier concentration $n$ for three different substrate orientations. The solid line gives the theoretical mobility at a compensation ratio $N_{\mathrm{A}}^{-} / N_{\mathrm{D}}^{+}=0.3 . T_{\mathrm{G}}=700^{\circ} \mathrm{C}, \mathrm{V} / \mathrm{III}=20$.

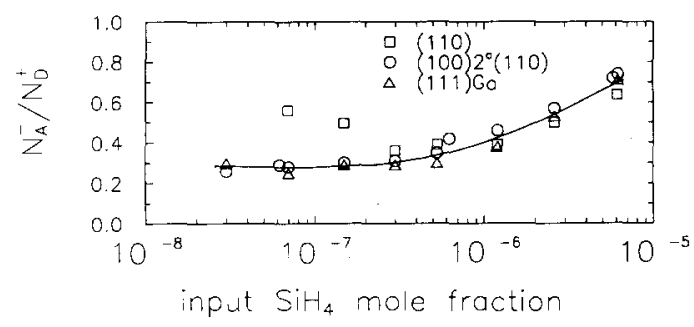

Fig. 4. Compensation ratio $\left(N_{\mathrm{A}}^{-} / N_{\mathrm{D}}^{+}\right)$versus silane input mole fraction for three different substrate orientations. The line in the figure is a guide to the eye.

pointed out that the calculations in refs. $[16,17]$ are based on dilute solid solutions so that our calculated values above a carrier concentration of $3 \times 10^{18} \mathrm{~cm}^{-3}$ should be treated with some care. The calculated results on $N_{\mathrm{A}}^{-} / N_{\mathrm{D}}^{+}$are given in fig. 4. For $\mathrm{SiH}_{4}$ mole fractions below $3 \times 10^{-7}$, the compensation ratio has a nearly constant value of 0.3 for the $(100) 2^{\circ}(110)$ and $(111) \mathrm{Ga}$ orientations. For the (110) orientation, however, the compensation ratio starts at a value 0.6 for low $P_{\mathrm{SiH}_{4}}$, decreasing to the value 0.3 for $P_{\mathrm{SiH}_{4}} \approx 3 \times 10^{-7}$. The rather high overall compensation is in contrast with other reports where the compensation ratio is found to be very low [18] or assumed to be zero $[5,6]$. We have checked whether the high $\mathrm{Si}_{\mathrm{As}}$ concentration could be due to a low effective $\mathrm{V} /$ III ratio during growth. This possibility exists in our reactor, because, despite the high V/III ratio used in our experiments, during growth an arsenic deposit is formed at the cooled top wall of the reactor thereby lowering the effective V/III ratio. Removing of the top cooling, whereby also the arsenic deposition was avoided, resulted in the same compensation ratios, however.

For carrier concentrations larger than $3 \times 10^{18}$ $\mathrm{cm}^{-3}\left(P_{\mathrm{SiH}_{4}}>10^{-6}\right)$ all compensation ratios rise from the value 0.3 to 0.7 at $P_{\mathrm{SiH}_{4}}=6 \times 10^{-6}$. This means that at higher silicon concentrations more $\mathrm{Si}$ atoms are incorporated at As-sites.

The relatively high compensation of 0.3 found in this study in the lower dopant regions may be due to a difference in growth conditions. We are growing layers in the region where the gas flow and the temperature profiles are fully developed and stabilized, whereas most experiments which 
are mentioned in literature have been performed with a very short susceptor which is placed in the region where both gas flow and temperature profiles still have to develop. The consequences are that in our reactor surface equilibrium processes can get a chance to adjust, this in contrary to the short cells where kinetics will prevail. The existence of silicon on As-sites could be proved by the presence of a $\mathrm{Si}_{\mathrm{As}}$ peak in photoluminescence spectra. Our PL spectra clearly reveal a $\left(e, A^{0}\right)$ transition, in which $\mathrm{Si}_{\mathrm{As}}$ as an acceptor is involved, when $n$ is larger than $2 \times 10^{17} \mathrm{~cm}^{-3}$ (fig. 5). Below this value the $\mathrm{Si}_{\text {As }}$ peak may still be present, but is difficult to resolve due to the overlap with a $\mathrm{C}_{\mathrm{As}}$ peak. The fact that $\mathrm{Si}$ acts as an acceptor in $\mathrm{GaAs}$ is not a new phenomenon. Under LPE conditions, it is known that even $\mathrm{p}$-type GaAs can be obtained by adding $\mathrm{Si}$ to the Ga melt [19]. Also under MBE conditions, p-type GaAs can be grown on certain substrate orientations $[13,14]$. A detailed analysis of the compensation effect based on a step incorporation mechanism will be given in a separate paper [20].

The total carrier concentration $\left(N_{\mathrm{D}}^{+}+N_{\mathrm{A}}^{-}\right)$can be calculated based on the net carrier concentration and the mobility values. The calculated $N_{\mathrm{D}}^{+}$ $+N_{\mathrm{A}}^{-}$value is equal to the total number of incorporated $\mathrm{Si}$ atoms if the $\mathrm{Si}$ atoms are exclusively incorporated at lattice sites and completely ionized. This is not true when certain $\mathrm{Si}$ complexes, e.g.

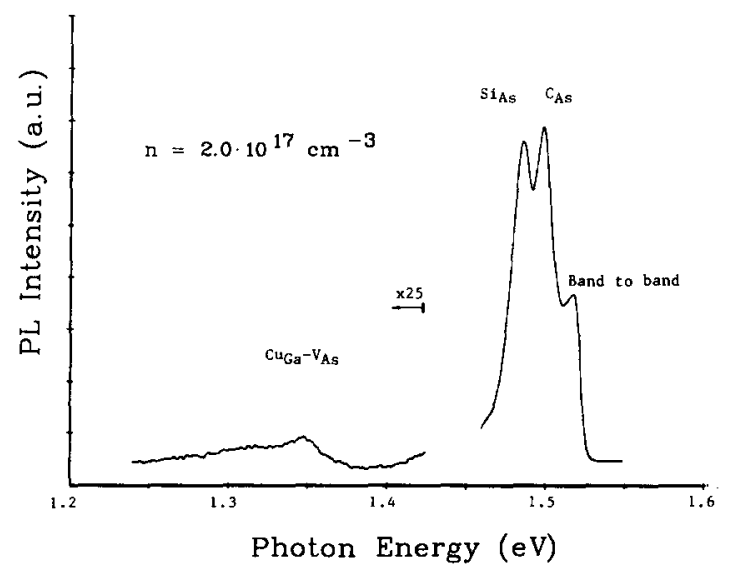

Fig. 5. Low temperature (5 K) PL spectrum for a (100) $2^{\circ}(110)$ sample with a carrier concentration $n=2 \times 10^{17} \mathrm{~cm}^{-3}$. The (e, $\mathrm{Si}_{\text {As }}$ ) peak is clearly resolved.

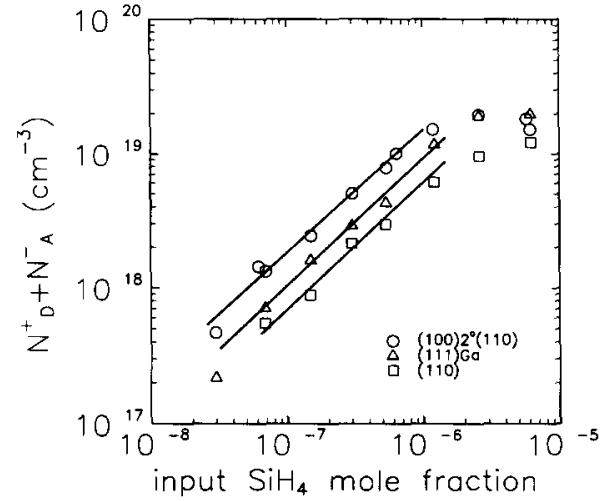

Fig. 6. Total carrier concentration $N_{\mathrm{D}}^{+}+N_{\mathrm{A}}^{-}$versus the input mole fraction of silane for three different substrate orientations. $T_{\mathrm{G}}=700^{\circ} \mathrm{C}, \mathrm{V} / \mathrm{III}=20$.

$\left[\mathrm{Si}_{\mathrm{Ga}}-\mathrm{V}_{\mathrm{Ga}}\right]$, are formed as is characterized by infrared local vibration mode (LVM) absorption [21]. However this $\left[\mathrm{Si}_{\mathrm{Ga}}-\mathrm{V}_{\mathrm{Ga}}\right]$ complex was not observed for a sample doped up to $1 \times 10^{18} \mathrm{~cm}^{-3}$ so that our assumption is quite probable. In fig. 6 the $N_{\mathrm{D}}^{+}+N_{\mathrm{A}}^{-}$values are given versus the input of silane for the three orientations. They again show the earlier observed systematic trend $(100) 2^{\circ}(110)$ $>(111) \mathrm{Ga}>(110)$, despite the difference in mobilities found for these orientations. The GaAs growth rate was found to be constant for all the orientations studied, so that its influence on the $\mathrm{Si}$ incorporation can be excluded.

The difference in the total amount of $\mathrm{Si}$ found in various substrate orientations must originate from processes such as diffusion in the gas phase, adsorption-desorption over the surface, and diffusion of the adsorbed species on the surface. The selective incorporation of $\mathrm{Si}$ on an arsenic or a gallium position only can take place during the incorporation at a step or a kink site. Incorporation means that double or triple bonds are formed so that desorption is unlikely once the silicon atom is arrived at the step or kink site. Because all orientations receive the same silicon flux in the form of $\mathrm{SiH}_{2}$ radicals (viz. those radicals which are produced in the chemical boundary layer within its diffusion length from the substrate [3]), all surfaces in principle could have the same coverage of silicon species. The differences in incorporation rates in our experiments, indicate that 
the difference in the sticking coefficient or the difference in partial desorption must play a role. In other words, a partial equilibrium must be present at these surfaces. The origin for the difference in adsorption-desorption in principle can be found in the absolute value for the bond strength for the $\mathrm{SiH}_{2}$ radical with the adsorption sites on the (100), (111)Ga and (110) surfaces. Under MOCVD conditions the (100) surface is arsenic stabilized, where all arsenic atoms form dimers with each other in a $\sigma-\pi$ configuration. No extra $\mathrm{AsH}_{3}$ adsorption will be possible on this arsenic stabilized surface. Adsorption of the electronically incomplete shell species $\mathrm{SiH}_{2}$ on an arsenic atom is favoured, however, because the electron shell becomes completely filled in this process. The (111)Ga surface, on the other hand, is electronically poor because only single dangling bonds are present on the surface. The arsenic-rich conditions now do not lead to an arsenic stabilized surface, but only result in adsorption of $\mathrm{AsH}_{3}$. So the great difference with the (100) surface is that the $\mathrm{AsH}_{3}$ molecules on the (111) Ga surface do not form dimers, i.e. do not form an intrinsic part of the lattice. Adsorption of $\mathrm{SiH}_{2}$ on the (111)Ga surface will occur at the gallium sites which are left free. Because of the electron deficiency of the $\mathrm{Ga}$ atoms, an incompletely filled adsorption bond will be formed between $\mathrm{SiH}_{2}$ and $\mathrm{Ga}$. Because of the electron deficiency, combined with the electropositive character of $\mathrm{Ga}$ with respect to arsenic, the $\mathrm{Si}-\mathrm{Ga}$ bond strength will be smaller than the $\mathrm{Si}-\mathrm{As}$ bond strength. The consequence is that desorption of $\mathrm{SiH}_{2}$ on a (111) Ga surface is somewhat larger than on a (100) surface in the As-stabilized configuration. Because the step incorporation chances for both surfaces are alike, as well as the diffusion possibilities over the surface, the total incorporation rate is mainly determined by the silicon coverage on these surfaces. One can say that these adsorbed silicon species form the supply source to the steps. The consequence is that the silicon incorporation rate on (100) is larger than on (111)Ga. This will be discussed in more detail in a forthcoming paper [20].

The still smaller incorporation rate on (110) has to be explained by the zipper-like growth along

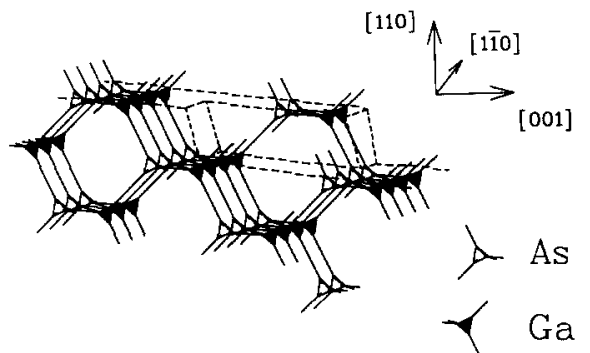

Fig. 7. Side-view of a (110) surface showing the [001] steps and a kink site. The periodic bond chains are lying along the channels in the $[1 \overline{1} 0]$ direction.

the channels in the $[1 \overline{1} 0]$ directions, where the periodic bond chains are lying [22,23] (fig. 7). Not only steric hindrance now plays an important role as to what diminishes the sticking coefficient, but also the difference in diffusion behaviour which is almost only restricted to a linear motion along the channel line.

The above discussion, although difficult to quantify, gives the trend for the incorporation of Si on the various orientations, viz. $(100)>(111) \mathrm{Ga}$ $>(110)$, as is found experimentally.

\subsection{Silicon precipitation}

For carrier concentrations higher than $3 \times 10^{18}$ $\mathrm{cm}^{-3}$ saturation occurs for all the substrate orientations studied (fig. 1). The saturation point corresponds to a silane input mole fraction of about $1 \times 10^{-6}$.

When the silicon concentration in GaAs is increased, the solubility limit of $\mathrm{Si}$ in GaAs can be exceeded. The solubility of $\mathrm{Si}$ in solid GaAs has been determined to reach a maximum of 4 at\% $\left(\sim 1.6 \times 10^{21} \mathrm{~cm}^{-3}\right)$ at the eutectic temperature of $1130^{\circ} \mathrm{C}$ [24]. However more precise data which cover the lower temperature region used for MOCVD $\left(550-750^{\circ} \mathrm{C}\right)$ are lacking so that no prediction can be given when precipitation will occur at these temperatures.

One can proceed in another way by calculating the chemical composition of the $\mathrm{Si}-\mathrm{Ga}-\mathrm{As}-\mathrm{H}-\mathrm{C}$ system using the existing data base of chemical equilibrium constants. For the growth temperature of $1000 \mathrm{~K}$ of $\mathrm{GaAs}$ by MOCVD, equilibrium 


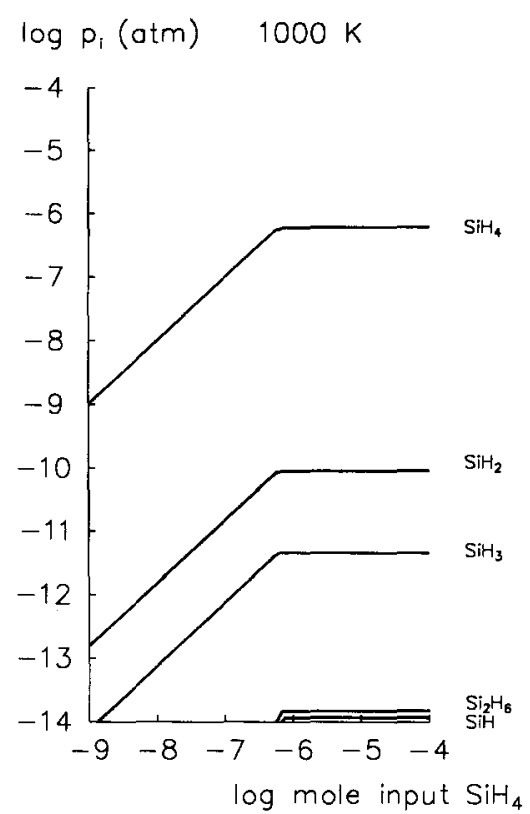

Fig. 8. Gas phase partial pressures of silicon compounds as a function of the input mole fraction of silane as calculated from thermodynamic equilibrium constants. The system contains TMG, $\mathrm{AsH}_{3}$, and $\mathrm{SiH}_{4}$ in $\mathrm{H}_{2}$ at 1 atm total pressure and at a temperature of $1000 \mathrm{~K}$. All the non-silicon containing species have been omitted in this figure. The horizontal parts in the curves are due to the formation of solid silicon which fixes the pressures.

calculations have been performed as a function of the input mole fraction of silane [25].

For input values of $\mathrm{SiH}_{4}$ larger than $1 \times 10^{-6}$ thermodynamics predicts the formation of solid silicon (fig. 8). Above this value the partial pressures of all gaseous silicon species are fixed, because all the additional silicon which is introduced into the system will be transferred into solid silicon.

The theoretically calculated value of input silane mole fraction of $1 \times 10^{-6}$ where silicon nucleation will occur, favourable coincides with the observed tendency for electrical saturation (fig. 1). It must be understood that differences will exist between the incorporation behaviour, which is a kinetic process, and the theoretically predicted equilibrium situation. When precipitation is predicted, it will only occur in practice when the silicon atoms in the bulk of the crystal have time enough to find each other (larger diffusion coefficients at the given temperature together with low growth rates). When the growth temperature is low enough, as in MBE systems, the individual silicon atoms may simply be trapped in the grown layers and never will have a chance to come to equilibrium because of the small diffusion coefficient in the bulk. Such a system will be supersaturated and will show a large domain of electrical activity as indeed has been observed [26,27]. In MOCVD the temperatures are higher and consequently the silicon atoms will have more chance to meet each other during the growth process. This promotes clustering and consequently a smaller electric active region is to be expected. This also is found experimentally.

The Si nucleation has been confirmed indirectly by photo-luminescence (PL) experiments. By looking at the band to band recombination peak in the PL spectra, it is found that when the carrier concentration is above a certain value, which in our case is approximately $1 \times 10^{17} \mathrm{~cm}^{-3}$, the peak position starts to shift to higher energies (fig. 9). This process is caused by the filling of the conduction band [28]. The peak energy keeps increasing when more $\mathrm{Si}$ is incorporated at lattice sites. This result is also found by Druminski et al. [6]. An interesting point from their study is that when the total Si concentration is higher than $1 \times 10^{19} \mathrm{~cm}^{-3}$, the PL peak position is fixed. We were unable to confirm these results because the samples with the highest $\mathrm{Si}$ content showed no photoluminescence signal at all. The results of Druminski et al. show that the excess $\mathrm{Si}$ atoms are no longer electrically active as donors or acceptors. This phenomenon has been explained by the formation of precipi-

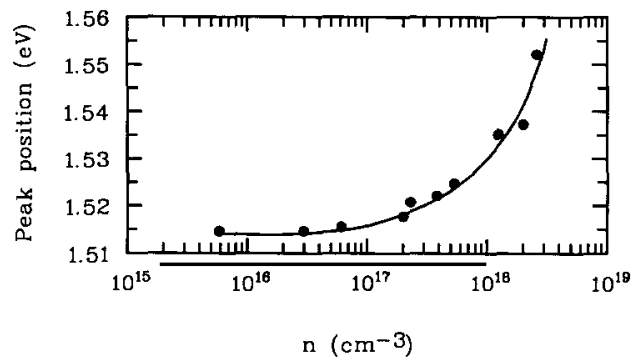

Fig. 9. PL peak position of the band to band recombination of silicon doped GaAs (at $5 \mathrm{~K}$ ) versus room temperature net carrier concentration. 
tates of $\mathrm{Si}$ or some other kind of non electrically active complex. Up to now no direct proof has been given of precipitate formation of $\mathrm{Si}$ in MOCVD GaAs.

There are some additional techniques which might be able to give information on precipitate formation, viz. SIMS, wet chemical etching, morphological study of the epitaxial layers and TEM. The SIMS technique is only suited to measure the total $\mathrm{Si}$ concentration, it can not discriminate whether the $\mathrm{Si}$ atoms are incorporated at the $\mathrm{Ga}$ or As lattice sites or incorporated as precipitates. Also the wet chemical etching technique DSL (defect selective etching with light) which has proved to be so powerful for defect revealing [29], fails, because at these high carrier concentrations the etch rate under illumination is simply zero, probably due to the high recombination rate of the created hole-electron pairs. Observation of the surface morphology in principle should give valuable results. In order to study this more precisely, an additional series of samples has been grown with $\mathrm{SiH}_{4}$ input mole fractions around the critical value $1 \times 10^{-6}$, viz. $6.2 \times 10^{-8}, 6.3 \times 10^{-7}$ and 5.8 $\times 10^{-6}$, respectively. The GaAs substrates used had three different orientations, (100), $(100) 2^{\circ}(110)$ and $(100) 4^{\circ}(110)$. The morphology appears to be similar for all the samples grown with the two lower $\mathrm{Si}$ input concentrations. For the highest $\mathrm{Si}$ input concentration, the morphology of the exact (100) samples show a difference as compared with the misoriented (100) samples (fig. 10). The surfaces in figs. 10a and 10b are flat, fig. 10c shows a wavy like surface consisting of macrosteps. In our opinion, the observed macrostep formation has to be explained as a result of step blocking by precipitates or clusters of adsorbed impurities.

Crystal growth is taking place by step propagation on the surface. The steps or the kink sites along a step provide favourite places for species to incorporate. According to the theory of Cabrera and Vermileya [30], a step which moves along an atomically smooth terrace will be stopped by a pair of impurities that are less than $2 \rho_{\mathrm{c}}$ apart from each other ( $\rho_{c}$ is the critical radius) and will squeeze itself between pairs of impurities when they are more than $2 \rho_{\mathrm{c}}$ apart. When a step is stopped by impurities, a macrostep has to be formed to override the impurities. This is true for immobile impurities. In case the adsorbed impurity atoms are still mobile and can diffuse over the surface, the moving step is able to drive the impurity atoms in front of the step. The larger the terrace is - as is the case for exact (100) - the higher the impurity density can become. Because of this condensation effect the critical distance between two impurities will be reached first at the
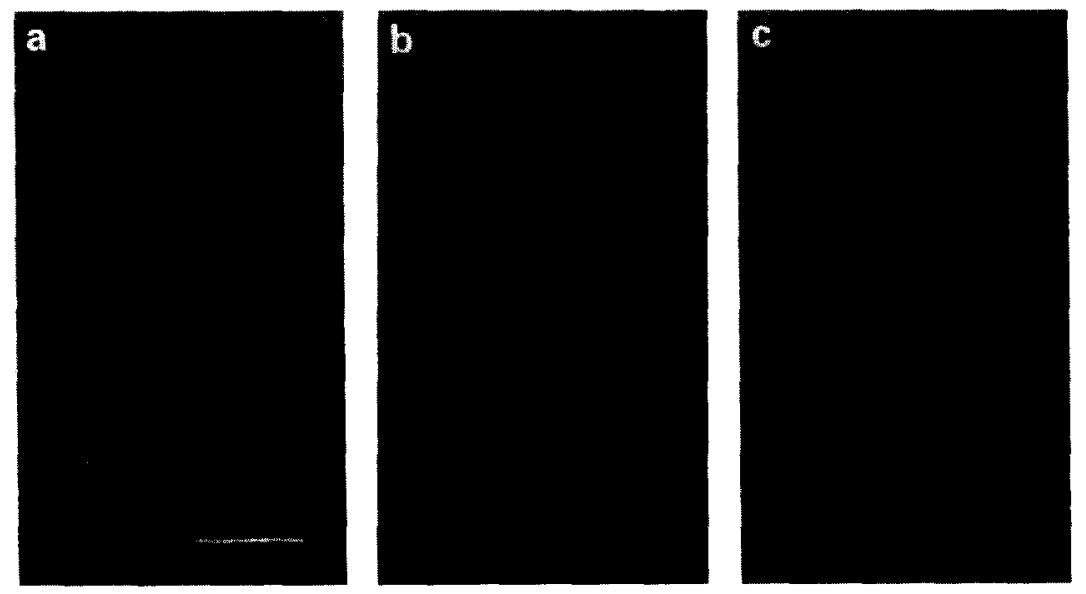

Fig. 10. Surface morphologies of epitaxial layers as obtained by interference microscopy for three (100) (mis-)oriented samples. The silane input mole fraction in each case was $5.8 \times 10^{-6}$. (a). (100) $4^{\circ}$ off towards $(110)$, (b) (100) $2^{\circ}$ off towards (110), (c). exact (100). At lower silane input values all surfaces are smooth. Marker represents $50 \mu \mathrm{m}$. 

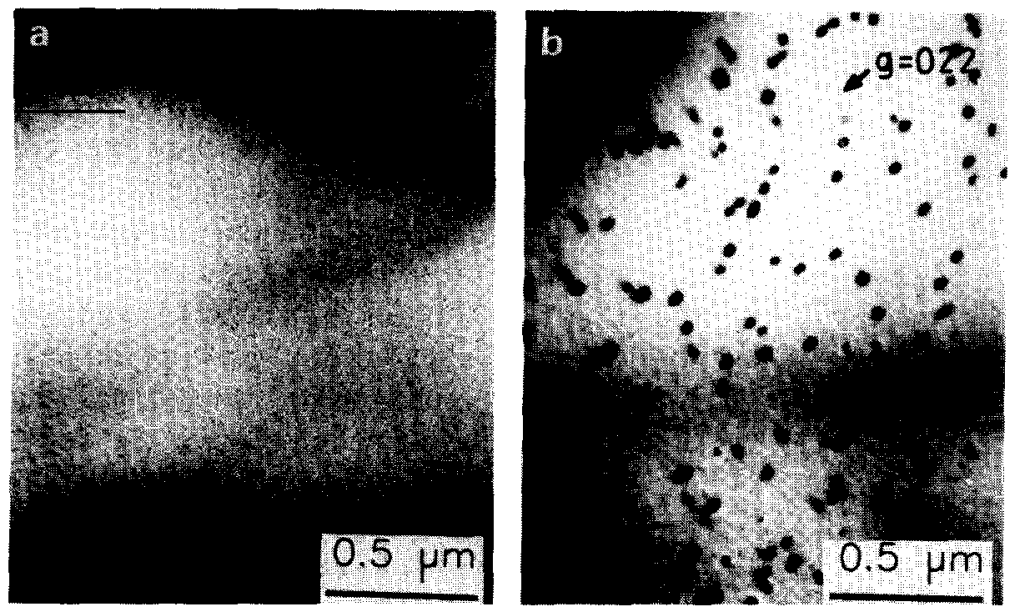

Fig. 11. TEM micrographs of two Si-doped samples: (a) silane mole fraction $6.2 \times 10^{-8}$ and (b) silane mole fraction $5.8 \times 10^{-6}$. The small granular contrast in the background is caused by surface damage due to ion-beam thinning.

exact orientation. This explains the difference in morphology between exact and misoriented (100) samples. The phenomenon of macrostep formation is often observed in solution grown crystals [31].

The most direct proof of the presence of precipitates has come from our TEM studies of these samples. Defects indeed have been observed in the samples grown with the highest silane input (fig. 11). These defects are identified as dislocation loops, interpreted as interstitial dislocation loops due to the Si-doping. Similar observations have been reported in studies of heavily Si-doped Bridgman-grown GaAs [32] and annealed Si-implanted GaAs [33]. The dislocation loop densities have been measured to be approximately $3 \times 10^{13}$ $\mathrm{cm}^{-3}$.

From the above we conclude that indeed precipitates are formed which cause the formation of macrosteps as well as the observed dislocation loops by TEM. It proves that Si nucleation starts between a $\mathrm{SiH}_{4}$ input mole fraction of $6.3 \times 10^{-7}$ and $5.8 \times 10^{-6}$. This is consistent with the thermodynamic analysis which predicts a critical $\mathrm{SiH}_{4}$ input mole fraction of $1 \times 10^{-6}$ for the formation of solid silicon. As these silicon precipitates will not contribute to the free carriers, the formation of precipitates consequently will decrease the electron mobility as is shown in fig. 3 .

\subsection{Rate limiting step of the silicon incorporation process}

It is known from previous studies [7] that the growth of GaAs by MOCVD at high temperature is limited by the diffusion of TMG through the gas phase. As a consequence gas phase depletion is known to occur, especially for long deposition lengths without a tilt of the susceptor. This depletion phenomenon can be calculated with a rather high precision $[7,34]$. In this study the depletion of the gallium containing gaseous species from the gas phase is demonstrated by the exponential decrease in growth rate as given in fig. 12a. If the silicon incorporation process also would be dominated by gas phase diffusion, a similar depletion behaviour has to be expected for the Si doping as well. However, when the $\mathrm{Si}$ incorporation is corrected for the difference in growth rate and a small difference in growth temperature over the susceptor, there appears to be no depletion at all (fig. 12b). The corrections are based on the observations (i) that the $\mathrm{Si}$ incorporation rate is inversely proportional to the GaAs growth rate, which is also reported by other authors $[9,35,36]$, and (ii) that the $\mathrm{Si}$ incorporation rate from silane is a strong function of the growth temperature 

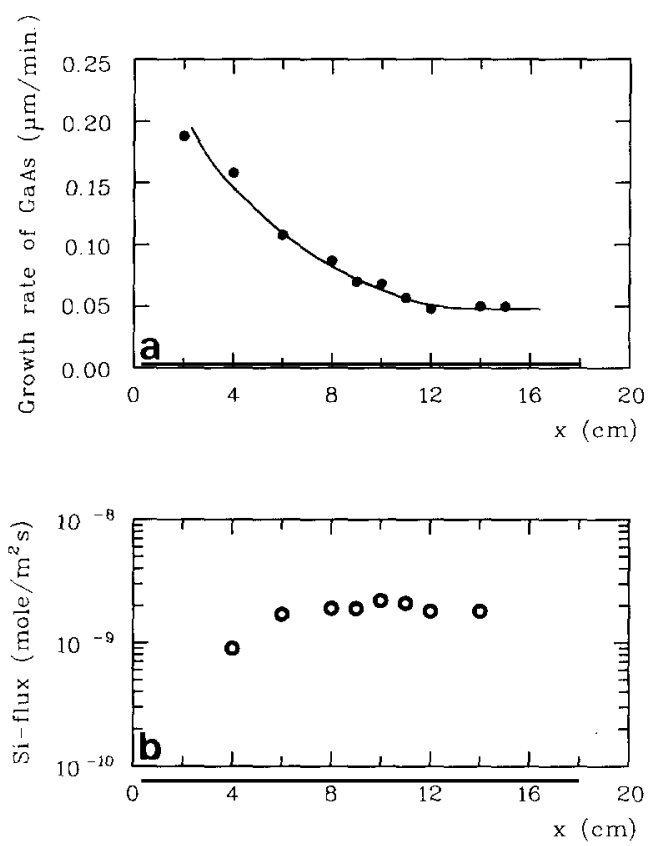

Fig. 12. (a) Growth rate of GaAs as a function of the axial position $x$ in the reactor, showing the exponential depletion effect. (b) Silicon flux $\left(\mathrm{mol} / \mathrm{m}^{2} \cdot \mathrm{s}\right)$ to the surface as a function of the axial position $x$. The flow and temperature profiles are fully developed for $x \geq 6 \mathrm{~cm}$.

$[1,9,36]$,

$n \propto \mathrm{e}^{-E_{\mathrm{a}} / R T}$,

where $E_{\mathrm{a}}$ is the activation energy for the incorporation process.

In a separate paper we will report on the $\mathrm{Si}$ incorporation rate as function of the growth temperature [3], the activation energy for this process is found to be $47 \mathrm{kcal} / \mathrm{mol}$. This high value corresponds to the activation energy $E_{\mathrm{a}}\left(k_{+}\right)$of the decomposition reaction of silane according to

$\mathrm{SiH}_{4} \stackrel{k_{+}}{\rightarrow} \mathrm{SiH}_{2}+\mathrm{H}_{2}$.

The product $\mathrm{SiH}_{2}$ is believed to adsorb on the GaAs surface and to be responsible for the incorporation of $\mathrm{Si}$. Due to the high activation energy this decomposition reaction will only occur in appreciable quantities at the highest gas temperatures, i.e. close to the crystal surface in the chemical boundary layer [3]. Because the activation energy $E_{\mathrm{a}}\left(k_{-}\right)$for the back reaction is very small
$\left(E_{\mathrm{a}}\left(k_{-}\right) \ll E_{\mathrm{a}}\left(k_{+}\right)\right)$, the diffusion length of the $\mathrm{SiH}_{2}$ radicals in the hydrogen carrier gas of 1 bar is extremely small, viz. about $30 \mu \mathrm{m}$ [3], so only those $\mathrm{SiH}_{2}$ molecules that are created very close to the hot susceptor i.e. in the lowest part of the chemical boundary layer can be incorporated. $\mathrm{SiH}_{2}$ molecules that are created away from the susceptor will react with $\mathrm{H}_{2}$ and form $\mathrm{SiH}_{4}$ again. This phenomenon explains the nearly constant $\mathrm{Si}$ concentration in the bulk gas phase and the absence of depletion. So for the doping of GaAs with $\mathrm{Si}$ using silane, the decomposition of $\mathrm{SiH}_{4}$ into $\mathrm{SiH}_{2}$ very close to the surface followed by diffusion of $\mathrm{SiH}_{2}$ towards the crystal surface is the rate limiting step for the incorporation process, although the growth process itself is diffusion limited.

\section{Conclusion}

Doping studies have been carried out as a function of silane input fraction over the entire dopant range of silicon in GaAs. It is found that up to a carrier concentration of $3 \times 10^{18} \mathrm{~cm}^{-3}$, the silicon incorporation varies linearly with the silane input concentration. The efficiency of the incorporation increases in the order $(100) 2^{\circ}(110)>$ (111) Ga $>$ (110). This trend can be explained by the relative bond strengths between $\mathrm{SiH}_{2}$ species and the various oriented surfaces, i.e. the difference in desorption energy. This implies that a certain degree of dopant adsorption-desorption equilibrium is present at these surfaces. For the (110) face in addition the size effect of the $\mathrm{SiH}_{2}$ molecules has to be taken into account.

All the Si-doped layers grown are found to be compensated. For the $(100) 2^{\circ}(110)$ and $(111) \mathrm{Ga}$ orientations, the compensation ratio has a value of about 0.3 for doping up to $3 \times 10^{18} \mathrm{~cm}^{-3}$, and increases above this value to 0.7 . The $(110)$ orientation always shows a higher compensation ratio.

For carrier concentrations larger than $3 \times 10^{18}$ $\mathrm{cm}^{-3}\left(P_{\mathrm{SiH}_{4}}>10^{-6}\right)$, precipitates in the crystal are formed due to $\mathrm{Si}$ nucleation in the matrix. This is in agreement with equilibrium calculations of the system $\mathrm{Si}-\mathrm{Ga}-\mathrm{As}-\mathrm{H}-\mathrm{C}$.

The rate limiting step for the $\mathrm{Si}$ incorporation is concluded to be the decomposition of $\mathrm{SiH}_{4}$ into 
$\mathrm{SiH}_{2}$ and $\mathrm{H}_{2}$ followed by diffusion of $\mathrm{SiH}_{2}$ to the surface. This process happens very close to the hot substrate because of the very small free diffusion length of the $\mathrm{SiH}_{2}$ radical in 1 bar $\mathrm{H}_{2}$.

\section{Acknowledgments}

This work was performed as a part of the research program of the "Stichting voor de Technische Wetenschappen" (STW) with financial support from the "Nederlandse Organisatie voor Wetenschappelijk Onderzoek" (NWO).

\section{References}

[1] T.F. Kuech, E. Veuhoff and B.S. Meyerson, J. Crystal Growth 68 (1984) 48.

[2] E. Veuhoff, T.F. Kuech and B.S. Meyerson, J. Electrochem. Soc., Solid-State Sci. Technol. 132 (1985) 1958.

[3] P.R. Hageman, X. Tang, M.H.J.M. de Croon and L.J. Giling, J. Crystal Growth 98 (1989) 249.

[4] M. Shimazu, K. Kamon, K. Kimura, M. Mashiia, M. Mihara and M. Ishii, J. Crystal Growth 83 (1987) 327.

[5] R. Azoulay, L. Dugrand, D. Ankri and E.V.K. Rao, J. Crystal Growth 68 (1984) 453.

[6] M. Druminski, H.-D. Wolf, K.-H. Zschauer and K. Wittmaack, J. Crystal Growth 57 (1982) 318.

[7] J. van de Ven, G.M.J. Rutten, M.J. Raaijmakers and L.J. Giling, J. Crystal Growth 76 (1986) 352.

[8] L.M. Fraas, J.A. Cape, P.S. McLeod and L.D. Partain, J. Vacuum Sci. Technol. B3 (1985) 921.

[9] S.J. Bass, J. Crystal Growth 47 (1979) 613.

[10] K. Okamoto, S. Onozawa and T. Imai, J. Appl. Phys. 56 (1984) 2993.

[11] N. Bottka, R.S. Sillmon and W.F. Tseng, J. Crystal Growth 68 (1984) 54.

[12] T. Nakanisi, J. Crystal Growth 68 (1984) 282.

[13] S.S. Bose, B. Lee, M.H. Kim, G.E. Stillman and W.I. Wang, J. Appl. Phys. 63 (1988) 743.

[14] W.I. Wang, Surface Sci. 174 (1986) 31.
[15] S. Subbanna, H. Kroemer and J.L. Merz, J. Appl. Phys. 59 (1986) 488.

[16] W. Walukiewicz, L. Lagowski, L. Jastrzebski, M. Lichtensteiger and H.C. Gatos, J. Appl. Phys. 50 (1979) 899.

[17] W. Walukiewicz, J. Lagowski and H.C. Gatos, J. Appl. Phys. 53 (1982) 769.

[18] R. Venkatasubramanian, K. Patel and S.K. Ghandhi, J. Crystal Growth 94 (1989) 34.

[19] F.E. Rostztoczy, J. Electrochem. Soc. 17 (1968) 516.

[20] X. Tang, J. te Nijenhuis, Y. Li and L.J. Giling, to be published.

[21] J. Maguire, R. Murray, R.C. Newman, R.B. Beall and J.J. Harris, Appl. Phys. Letters 50 (1987) 516.

[22] L.J. Giling and W.J.P. van Enckevort, Surface Sci. 161 (1985) 567.

[23] W.J.P. van Enckevort and L.J. Giling, J. Crystal Growth 45 (1978) 90.

[24] L.A. Borisova and N.A. Valisheva, Izv. Akad. Nauk SSSR, Neorgan. Mater. 10 (1974) 1083.

[25] L.C. Keizer, X. Tang, R.Z.C. van Meerten and L.J. Giling, J. Crystal Growth, submitted.

[26] M. Heiblum, W.I. Wang, L.E. Osterling and V. Deline, J. Appl. Phys. 54 (1983) 6751.

[27] R. Sacks and H. Shen, Appl. Phys. Letters 47 (1985) 374.

[28] H.G.M. Lochs, X. Tang, F.L.M. Spijkers, H.G.M. Titulaer, R. Visser and L.J. Giling, to be published.

[29] J.L. Weyher and J. van de Ven, J. Crystal Growth 78 (1986) 191.

[30] N. Cabrera and D.A. Vermileya, in: Growth and Perfection of Crystals, Eds. R.H. Doremus, B.W. Roberts and D. Turnbull (Wiley, New York, 1958) p. 393.

[31] J.P. van der Eerden and H. Müller-Krumbhaar, Phys. Rev. Letters 57 (1986) 2431.

[32] D.B. Darby, P.D. Augustus, G.R. Booker and D.J. Stirland, J. Microscopy 118 (1980) 343.

[33] C.P. Stewart, R.t. Blunt, G.R. Booker and I.R. Sanders, Physica 116B (1983) 635.

[34] H. Moffat and K.F. Jensen, J. Crystal Growth 77 (1986) 108.

[35] R.J. Field and S.K. Ghandhi, J. Crystal Growth 74 (1986) 543.

[36] J.P. Duchemin, M. Bonnet, F. Koelsch and D. Huyghe, J. Electrochem. Soc., Electrochem. Sci. Technol. 126 (1979) 1134. 